\title{
Using the CAT for 3D Sketching in front of Large Displays
}

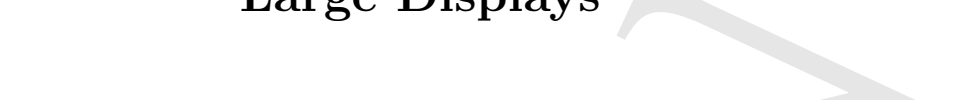

Hongxin Zhang ${ }^{1}$, Julien Hadim ${ }^{2}$, and Xavier Granier ${ }^{2}$

${ }^{1}$ State key Lab of CAD\&CG

Zhejiang University

Hangzhou 310027, PR China

zhx@cad.zju.edu.cn

2 INRIA Bordeaux Sud-Ouest - Universities of Bordeaux,

351, cours de la Libération

33405 Talence, France

\{hadim|granier\}@labri.fr

\begin{abstract}
Sketching, as an intuitive tool for creation and edition of $3 \mathrm{D}$ prototypes, is a topic of increasing interest to the community. These approaches are based on the natural ability of humans to quickly draw in $2 \mathrm{D}$ some characteristic curves of $3 \mathrm{D}$ objects. Unfortunately, some 3D modeling operations - like positioning different components - and the modeling in front of large displays have still not reached the same level of intuitively in sketching systems. The main difficulty is to leverage the intuitive 2D gesture abilities of humans and lift them to 3D operations. We present a new approach, based on a virtual 3D paper sheet metaphor and the use of a 6 degrees of freedom (DOF) device. With the associated interaction processes and visual feedbacks, it allows the user to quickly create and edit some sketched $3 \mathrm{D}$ models.
\end{abstract}

Key words: Large Display, Sketch-based 3D Modeling, Interaction Devices

\section{Motivation}

With the constant emergence and integration of new tools, current 3D modelers can become very complex to manipulate and can thus be very intimidating for non-expert users. Due to complicated operations and non-intuitive parameters, large training period are necessary for an user to be confident in 3D modeling . Creating 3D prototypes with mouse and keyboard only can thus become a slow and painful task. This problem has lead, during the recent years, to research and development of new approaches for simpler user interfaces. Some of the proposed solutions use the natural human ability to quickly sketch a global overview of an object. These approaches are naturally referred to as $3 D$ Sketching.

Research on 3D sketching has generated a new modeling approach: the system automatically infers a $3 \mathrm{D}$ shape according to a set of sketched $2 \mathrm{D}$ curves. Users can edit this model afterward to add details and to improve the geometric complexity with other advanced modeling operations (e.g., cutting and extrusion). All these operations are still based on sketching interactions. Since all 3D 
sketching systems use a drawing metaphor, they can be easily adopted even by non-experts.

Following the well-known sketching modeler "Teddy" [10], many solutions have been proposed for improving the surface generation $[11,2,20,18,6]$ and the editing interactions $[16,20,6]$. Most of these previous systems are based on a classic $2 \mathrm{D}$ pen-and-paper context, similar to the use of a drawing tablet. In general (e.g., $[10,2,20])$, the final model is obtained by combining different components resulting from $2 \mathrm{D}$ sketches on different planes. However, combining these different components requires switching between 2D sketching interactions and 3D manipulations (mainly positioning and rotation). Hence in our project, we want to develop an integrated approach for these two modes, resulting in a more intuitive mode-switch.

The main aim in our project is to provide an intuitive manipulation environment for $3 \mathrm{D}$ design in virtual reality centers. In these environments, several users can collaborate and share ideas for creating 3D prototypes in front of a large display. In order to ease the interaction, we propose to work with an interaction device that can provide a direct mapping between its physical orientation/position and the relative orientation/position of a 3D object on the screen. This direct mapping guarantees that any position can be easily recovered by any user.

Our work introduces the following contributions. First, we show how 3D sketching can be formulated by using a 3D metaphor of virtual 3D paper sheet similar to the canvas introduced by Dorsey et al. [7]. Secondly, we introduce a full system, based on this metaphor and the use of a device with six degrees of freedom (DOFs). Finally, we present the proposed modeling steps and interactions, combined with some visual cues in order to provide a user-friendly solution.

This paper will be presented as follows. We first describe the existing solution for sketching in the context of very large screens and some existing solutions for $3 \mathrm{D}$ positioning. We then describe our reference solution, and the associated interaction steps required for modeling and editing 3D prototypes. Before concluding and introducing some future work, we illustrate this solution by detailed modeling sessions and by providing some results.

\section{Previous Work}

Among previous sketching solutions, implicit surfaces $[11,2,20,1]$ are the most commonly used surface representation since they provide a simple tool for creating complex objects, due to their natural blending between different components. This blending still requires correctly choosing their relative 3D positions.

One solution to the 3D positioning problem is to remove the restriction of sketching only $2 \mathrm{D}$ curves, by providing an interaction to directly draw 3D curves with a tracked device. Lapides et al. [12] use a tablet mounted on a support that can be translated vertically. One hand is used of the $2 \mathrm{D}$ curve drawing and the other one for vertical translation of the tablet. Unfortunately, a good $3 \mathrm{D}$ visualization system is required for accurate 3D drawing; otherwise, these 
approaches can result in undesired solutions due to the differences between the visualization system and our spatial representation of a 3D world. Furthermore, these techniques demand acute coordination abilities, involving both hands.

It is usually easier to have some reference planes [21] or surfaces [8] to draw on. Tsang et al. [21] use a set on planes oriented along the main axes. Markosian et al. [14] use the projection of the 3D curves on the image plane and its projection on a reference plane (called a "shadow curve") for inferring the 3D shape. The sequence of interactions is inverted by Grossman et al. [8] to extend the reference plane to a reference surface generated by the "shadow curve". They use a "Tape Drawing" [3] approach for curve sketching.

More recently, approaches based on multiple reference planes have been extended by Dorsey et al. [7] for architectural design and analysis. Their system uses strokes and planar "canvases" as basic primitives like for a traditional sketchbook (one sketch for each canvas) but can not provide true free-form models.

For an improved kinesthetic feedback, some tracked two-handed interactions have been introduced. Sachs et al.[17] use two Polemus-tracked devices: a palette as the reference plane and a stylus of the sketching part. Thanks to the palette, $3 \mathrm{D}$ positioning of the $2 \mathrm{D}$ curve or $3 \mathrm{D}$ object is easily achieved. Schroering et al. [19] use the same approach with camera-tracked devices: a board as reference plane and a laser pointer as a stylus. Unfortunately, as for any hand-held device, this can be physically tiring for the user. Furthermore, when multiple users are involved in the modeling process, the direct matching between the devices and the $3 \mathrm{D}$ positioning is lost anytime when the devices are released, put back at their original position, or passed to an other user. In our project, we want to reduce this limitation in the context of $3 \mathrm{D}$ sketching.

\section{System Overview}

We introduce a virtual 3D paper sheet metaphor in our sketching system. We assume that the user draws different sketches on different reference planes and combines them together in order to create the final object [7]. For the combination, we extend the classical 2D desk to a 3D space. On the modeling side, we use convolution surfaces [20]. These surfaces inherit the advantages of implicit surfaces, being expressed as a simple combination of the different components. On the hardware side, our system uses the CAT [9] to solve the relative 3D positioning of the different paper sheets.

\subsection{Sketching using convolution surfaces}

In most sketching systems, everything begins with a $2 \mathrm{D}$ curve hand-drawn by users. Inheriting from the approach introduced by Teddy [10], a skeleton structure $[20,1]$ is extracted, based on the set of segments of the medial axis. A convolution with a linearly weighted kernel is then performed on each segment. By summing the fields of all segments, an analytical convolution surface is obtained. The resulting generic shape has a circular cross-section. New components 


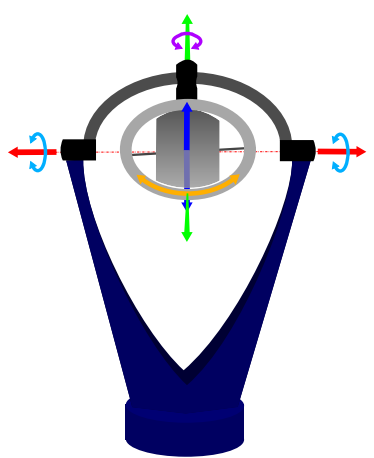

Synthetic view of the CAT

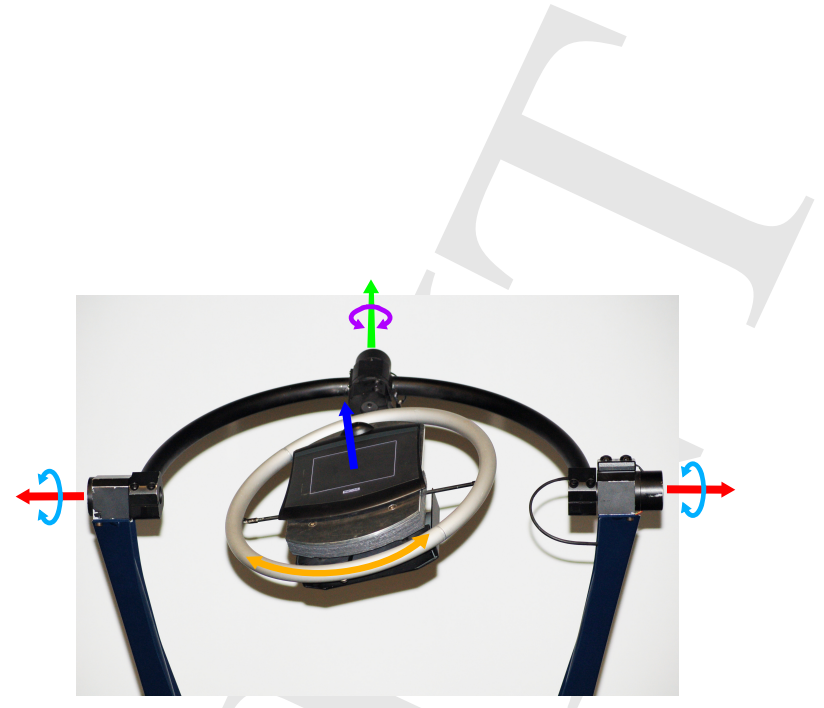

The real cat and its 6 DOFs

Fig. 1. Presenting the CAT and all 6 DOFs. The table can be rotated along all the 3 axes. Translations are detected by the user pressure along the 3 axes. Note the drawing tablet mounted on the table.

can be similarly designed by sketching on different projection planes, that we call $3 D$ virtual paper sheet for an interface point of view or reference plane for a modeling point of view. The convolution surface model smoothly combines the overlapping components. This approach overcomes several limitations of some sketched-based systems, including designing objects of arbitrary genus, objects with semi-sharp features, and the ability to easily generate a great variety of shapes.

On large displays, the quality of the resulting mesh is very important for displaying and for further manipulating the object. Hence regular topological connectivity and well sampled geometry are preferred. To achieve this goal, our current solution adopts the well-known polygonal tessellation - marching tetrahedra [4] - followed by a remeshing procedure [5] in order to smooth the final output.

\subsection{Introduction to the CAT interaction device}

Among the recent interaction devices, the CAT [9], a 6 DOFs interactor, provides us with a solution to the problems of 3D positioning. Compared to other devices, the CAT favors an unconstrained interaction since the user does not have to hold anything. Furthermore with the CAT, the rotations are directly controlled by an isotonic sensing mode while the (infinite) translations are controlled by an isometric sensing mode. Thanks to these features, it allows intuitive manipulations of $3 \mathrm{D}$ objects: the orientation of the table directly corresponds to the orientation of a plane in 3D space. Most similar devices like the SpaceMouse or 3D Mouse do not share such convenient properties. Our CAT-based solution can this simplify the orientation of the reference plane required for sketching. Furthermore, one one side, a translation is in theory an unbounded transformation and thus has to be relative. On the other side, a rotation is bounded and can thus be absolute. These interactions are naturally offered by the CAT. 


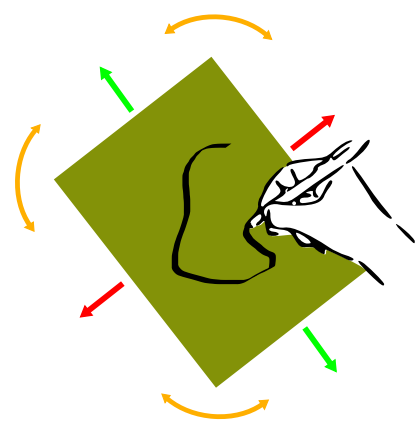

A paper sheet on a desk

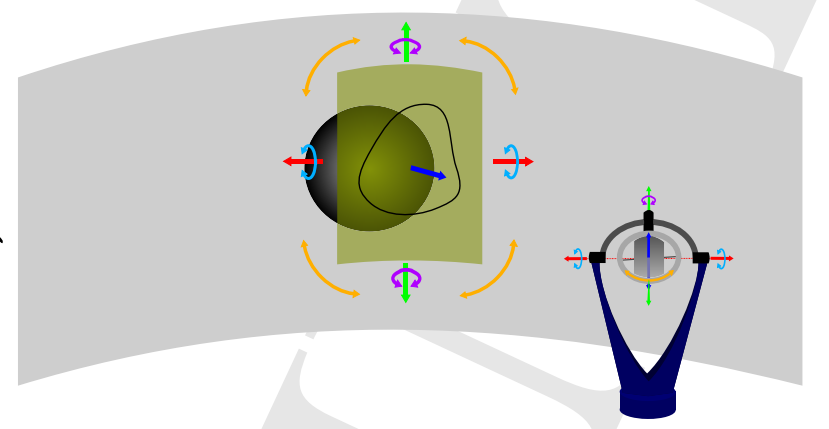

Virtual 3D paper sheet on a large display

Fig. 2. Movement of a real paper sheet on a desk (left) and the corresponding mapping between the rotations and the translations of the CAT and the movements of the virtual 3D paper sheet (right).

Moreover, a tablet fixed on the tabletop of the CAT is used for 2D interaction in our system. As required by most sketching solutions, a tablet can be directly mapped to a 3D virtual paper sheet, while keeping the user immersed in 3D environments (the $6 \mathrm{DOF}$ of the CAT). Therefore, all the interaction sequences have thus to be carefully designed in order to use this $6+2$ DOFs and will be described in the next section. With only one device, we can provide all the required DOFs.

\subsection{The virtual 3D paper sheet metaphor}

A sketching system is naturally based on a paper-and-pen metaphor. With the CAT, the tablet mounted on the top can be used as a reference plane, and thus directly associated to a $3 \mathrm{D}$ virtual paper sheet. The main problem for editing the different components of the object is thus to correctly position a set of reference planes in 3D (similar to the canvas in the work of Dorsey et al. [7]). Two main approaches can be used for this positioning: the traditional manipulation of the scene/object, or the manipulation of a virtual 3D paper sheet.

For the first one, user has to position the 3D scene/object relatively to a fixed 3D paper sheet. Unfortunately, it could be difficult to map the arbitrary shape of the resulting 3D object to the planar tabletop: the choice on the main orientation of the object corresponding to the orientation of the tabletop can be arbitrary.

On the CAT, the paper sheet can be directly associated with the tablet mounted on the moving table. We thus naturally decide to use the second approach: the positioning of the reference plane in 3D. The orientation of the table directly corresponds to the orientation of the 3D paper sheet (see Figure 2). The user just feels as if in front his desk, drawing multiple sketches on multiple paper sheets, and assembles them together. For the translations and rotations of the tablet are directly interpreted as the translation and the rotation of the virtual 3D paper sheet. 


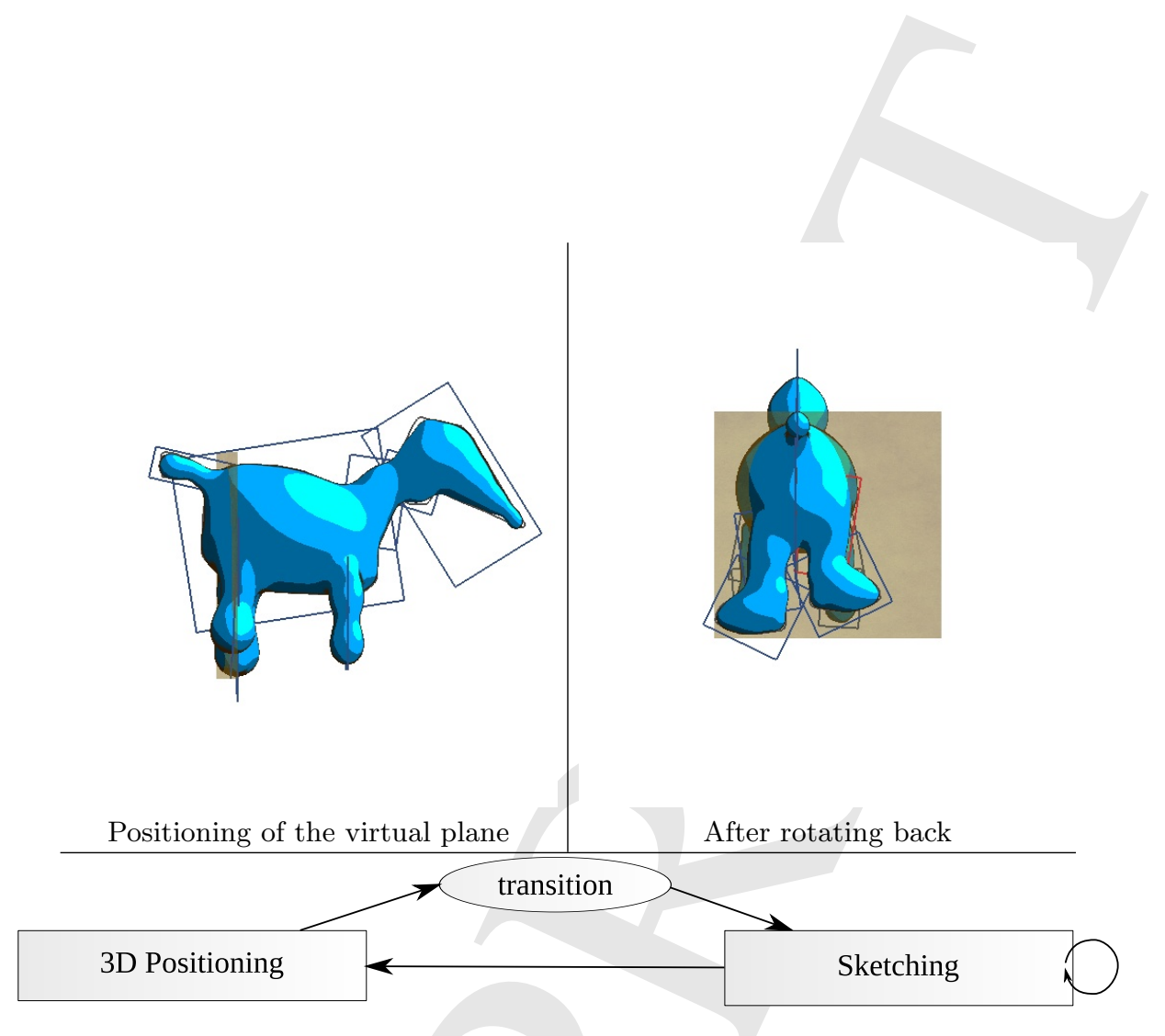

Fig. 3. Positioning of the virtual 3D paper sheet. The two modeling steps (upper images) and the associated interaction graph (lower image). During the 3D positioning mode, only the virtual plane is manipulated. During the sketching mode, the scene and the virtual 3D paper sheet are linked together. During the transition, users can rotate the virtual plane and the CAT to a horizontal configuration, more comfortable for drawing.

\section{Interactions for object creation}

The modeling session is generally broken down in two main steps (see Figure 3). During the first one, the user is moving the supporting plane relatively to the $3 \mathrm{D}$ scene. Once this virtual 3D paper sheet correctly positioned, the user has to change to sketching mode. Thanks to the CAT, these two steps are performed using a single device. In order to ease the positioning of the paper sheet relatively to the scene, the virtual 3D paper sheet is transparent.

For the positioning mode, only the virtual plane is controlled by the user. The direct mapping between the table orientation and the virtual 3D paper sheet simplifies this process.

For the sketching mode, the relative positions of the virtual 3D paper sheet and of the 3D scene are linked together: any translation or rotation of the CAT is directly applied to the scene and the virtual 3D paper sheet. This has two main advantages. First, if the tablet is moving during the sketching session, the relative positioning is not lost. Second, for a user point of view, it is easier to draw with the table at a horizontal position. This is not always the configuration after the positioning of the virtual 3D paper sheet. To finalize the transition to 


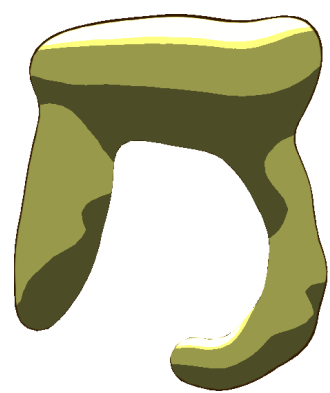

The complete object

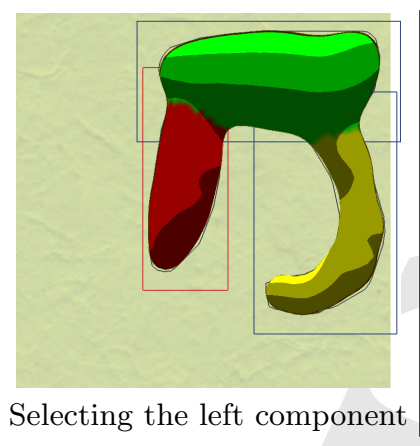

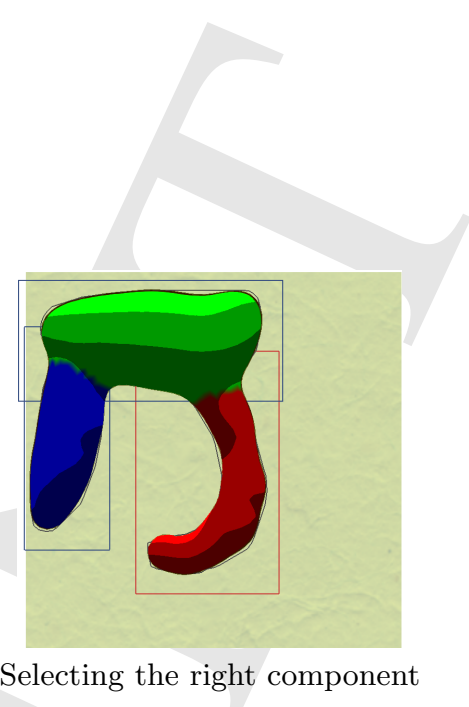

Selecting the right component

Fig. 4. Selection of a reference plane. In edit mode, each component has its own color and the selected component is highlighted in red. Note that we take the closest one to the center of the virtual 3D paper sheet.

the sketching mode, she thus has to manually rotate back at this position and - since the object and the virtual plane are linked together - the whole scene (i.e., the 3D object and the virtual plane) is rotated in order to move back also the virtual 3D paper sheet in the initial position.

Note that, during the transition from the positioning mode to the sketching mode, the whole scene is also translated in order to move the center of the virtual $3 \mathrm{D}$ paper sheet at the center of the screen. This guarantees that, if the rotation back is applied to the horizontal position, the virtual 3D paper sheet will be back at the initial orientation and also centered on the screen, in a configuration similar to the original one (see upper-left image in Figure 3).

Once the silhouette sketched, we save its association with the reference plane. A reference plane is defined by a center $c$ (the center of the silhouette) and a normal $\boldsymbol{n}$ (defined by the orientation of the virtual 3D paper sheet). We store also the corresponding 2D bounding box for display.

\section{Interactions for object edition}

Editing can be more complex. In the ideal case, when adding a new component to the final object, the previous 2-step approach is sufficient. To increase the freedom of the user, and the possibility of multi-users interaction, editing also needs to be supported. For most of the editing tasks, we need to retrieve the supporting plane corresponding to the component that we want to modify. This can be easily done once again using the CAT. The user moves the virtual plane in the 3D scene, and when it coarsely corresponds to the target plane, this one is selected. The user can thus return the table to the initial position (horizontal) and performs the 2D operations. Similarly to the modeling task, during this mode change, the whole scene is also rotated and translated in order to move back the virtual plane back to its original position.

The selection of the closest reference plane is done based on the plane orientation (i.e., normal $\boldsymbol{n}$ ) and its center $\boldsymbol{c}$. To this aim, we defined a weighted 


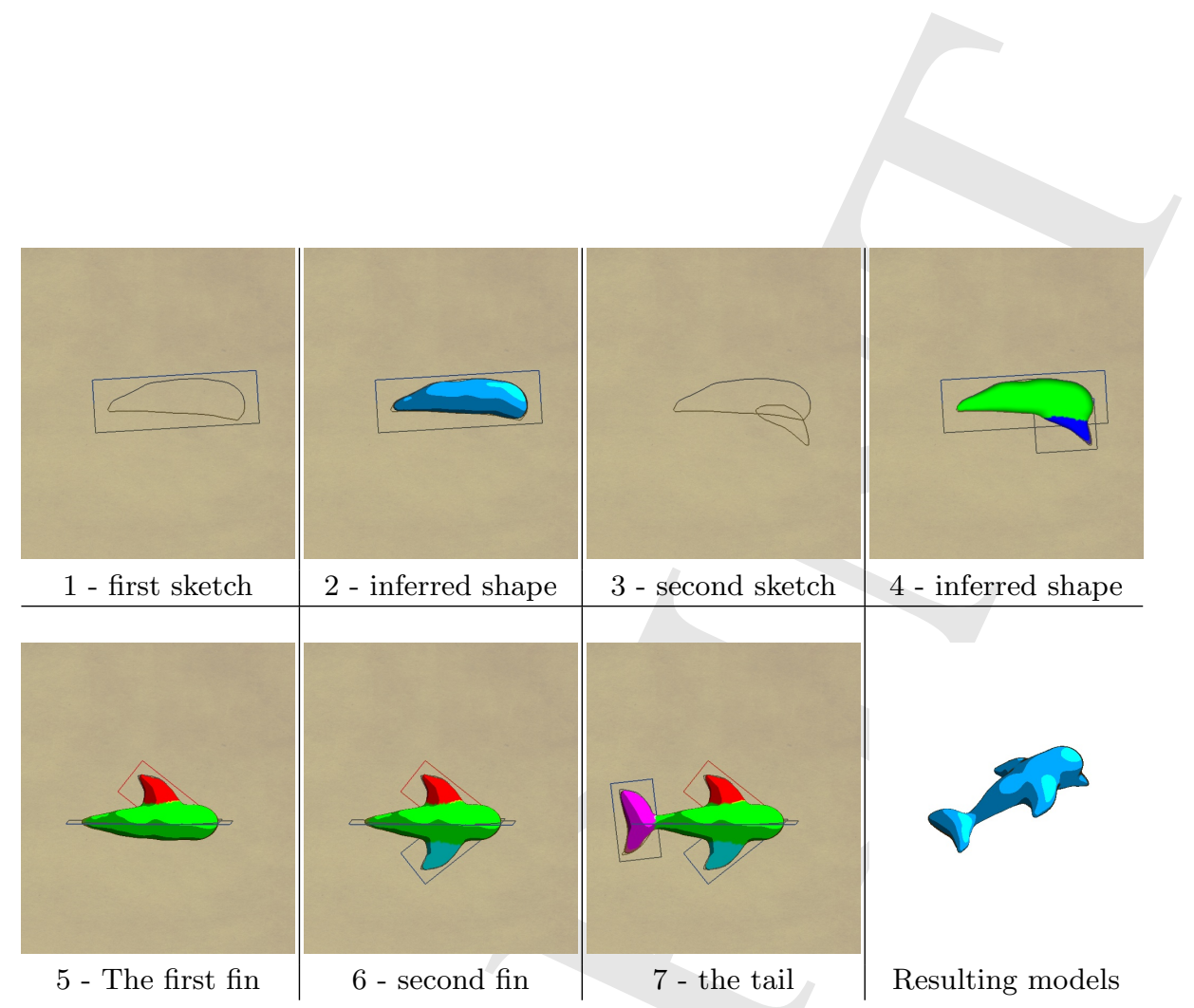

Fig. 5. Modeling a dolphin. The first four steps are done with nearly coplanar 3D paper sheet. During the transition from step 4 to step 5 , the CAT has been used to position the virtual plane orthogonally to dolphin's body, and rotated back as explained in Section 4 . The steps 5, 6 and 7 are now performed on the same plane

distance $d_{i}$, defined as

$$
d_{i}(\boldsymbol{n}, \boldsymbol{c})=\left(1-\left|\left\langle\boldsymbol{n}_{i} \cdot \boldsymbol{n}\right\rangle\right|\right)+\omega\left|\boldsymbol{c}_{i}-\boldsymbol{c}\right|,
$$

where $\boldsymbol{n}_{i}$ (resp. $\boldsymbol{c}_{i}$ ) denotes the normal (resp. center) of the existing reference plane $i$. The weight $\omega$ can be adjusted depending on the scene's dimensions ${ }^{1}$. Note that we use the absolute value of the cosine between the two normals to detect the proximity between two orientations, and that the positions are normalized to the unit bounding box.

In order to assist the user in selecting the correct reference plane corresponding to the object's component he whishes to modify, we use color cues. We associate to each component a color, which is used to display the reference plane and its corresponding surface (see Figure 4). We select a set of perceptually different colors, exclugind red, as we use this color to highlight the current closest plane.

\section{$6 \quad$ Modeling sessions}

To illustrate a typical modeling session with the presented approach, we review the steps involved in creating the shape of the dolphin in Figure 5. The user

\footnotetext{
${ }^{1}$ We use $\omega=0.1$ in our software, in order to give priority to the orientation.
} 


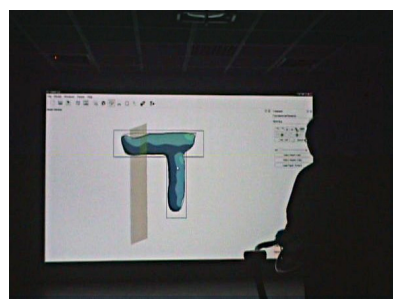

(a)

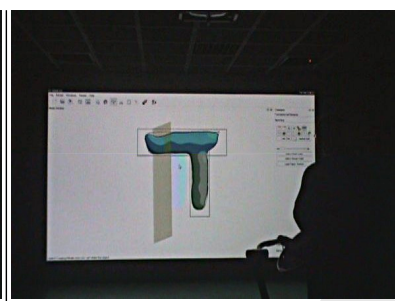

(b)

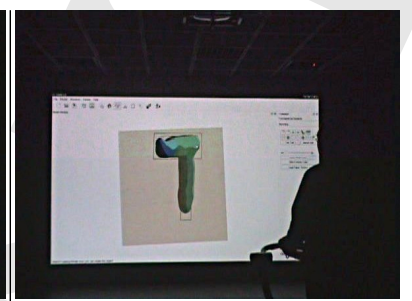

(c)

Fig. 6. 3D positioning of a new virtual plane. Once the position of the plane is validated by the user (a), a translation to the center is applied on both the plane and the object (b). The user can move back to horizontal position.

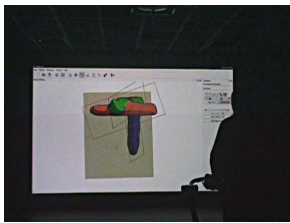

(a)

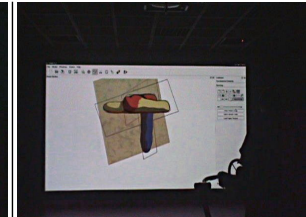

(b)

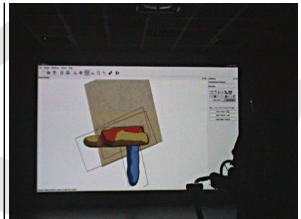

(c)

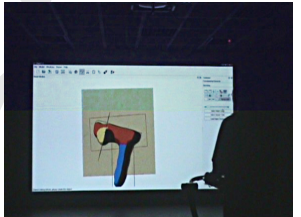

(d)

Fig. 7. Selecting an object component for editing. Beginning for the current position (a), the user moves the virtual paper sheet to select an other component (b). Each component has its own color, except for red which is used for selection. Once the selection is accepted by the user (c), a translation to the center is applied on both the plane and the object (d). The user can move back to horizontal position.

first draws the main body of the dolphin using two components (steps 1 to 4). To rotate the plane at a position orthogonal to the main body, the CAT table top has to be vertical. Once back in sketching mode, the rotation of the CAT is applied to the virtual paper sheet and to the objects: the user can thus move back the virtual paper sheet to a nearly horizontal configuration, more comfortable for sketching.

This transition is also illustrated ${ }^{2}$ in Figure 6 . Not that, once the position of the virtual plane corresponds to the user's whish, the transition to sketching mode results in a translation of the whole scene (object and plane) to the center of the screen. This translation allows the user to quickly bring back the virtual sheet in front of him: the only action required is the rotation back of the CAT. During the whole process, the orientation of the CAT table always corresponds to the orientation of the virtual paper sheet. This sequence of actions is similar when it comes to select an existing component (cf. Figure 7).

\footnotetext{
${ }^{2}$ The images are samples of the associated video. The difference in color between the upper and lower part is due to our stereo system.
} 


\section{Conclusion}

In this paper, we presented new interactions for 3D sketching in front of large displays using the CAT device. The philosophy of our system is to provide a comfortable alternative 3D prototyping interface for large displays, with the intention of avoiding the inherent interaction drawbacks of traditional mouse-andkeyboard-based interfaces. The proposed system enables users to share modeling ideas freely by simply drawing 3D sketch curves and manipulating the CAT. We use a virtual 3D paper sheet as a natural modeling metaphor for 3D sketching. Such a 3D paper sheet can be conveniently used to help users determine orientation and depth information while sketching 3D curves. Moreover, this 3D paper sheet can be also applied to select, move, rotate, and modify specific curves so as to help users to achieve the prototyping goal. As demonstrated in the result section, even non-expert users can use our free-form modeler without any difficulty thanks to its ergonomic and comfortable interface. More user study has to be done in order to confirm this assumption.

Based on the presented approach, there are several potential directions and improvement can be explored in the future. First, due to the drawbacks of implicit surface representations, we can only prototype relatively rough and smooth objects. Working on improvement of skeleton extraction $[1,13]$ could be helpful to enhance the modeling capabilities ability of the system. Second, we will focus on the specific modeling applications to take advantages of field prior knowledge, such as sketch-based architectural design which has been already explored by [7]. Third, similar to the approach of Nealen et al. [15], the CAT can be applied to select a section of the object by positioning a 2D plane. Therefore, it could be useful in the geometrical operations of extrusion, cutting, sharpening or smoothing the resulting profile-curve [15]. Moreover, it is worth noting that deformations of reference plane are affordable solution to enlarge the realm of prototyping shapes.

\section{Aknowledgement}

This project is supported by the French embassy in China and the Egid. This work is also supported in part by the National Basic 973 Program of China (Grant No. 2002CB312102), the National 863 Program of China (Grant No. 2006AA01Z335), and the National Natural Science Foundation of China (Grant No. 60505001). We would like to thank Immersion S.A.S. ${ }^{3}$ for the access to the CAT and to a Reality Center. We also want to greatly thank the anonymous reviewers for their comments and Jean-Sébastien Franco for proof-reading the final version.

\section{References}

1. Anca-Ileana Alexe, Loïc Barthe, Véronique Gaildrat, and Marie-Paule Cani. A sketch-based modelling system using convolution surfaces. Technical Report IRIT2005-17-R, IRIT, Université Paul Sabatier - Toulouse (France), July 2005.

\footnotetext{
3 http://www.immersion.fr
} 
2. B. De Araujo and J. Jorge. Blobmaker: Free-form modelling with variational implicit surfaces. In Proc. of "12th Encontro Português de Computação Grafica" (12th EPCG), pages 17-26, October 2003.

3. Ravin Balakrishnan, George Fitzmaurice, Gordon Kurtenbach, and William Buxton. Digital tape drawing. In UIST '99: Proc. annual symposium on User interface software and technology, pages 161-169. ACM, 1999.

4. Jules Bloomenthal. An implicit surface polygonizer. In Graphics Gems IV, pages 324-349. Academic Press, 1994.

5. Mario Botsch and Leif Kobbelt. A remeshing approach to multiresolution modeling. In SGP '04: Proc. Eurographics/ACM SIGGRAPH symposium on Geometry processing, pages 185-192. ACM, 2004.

6. Joseph Jacob Cherlin, Faramarz Samavati, Mario Costa Sousa, and Joaquim A. Jorge. Sketch-based modeling with few strokes. In SCCG '05: Proc. spring conference on Computer graphics, pages 137-145. ACM, 2005.

7. Julie Dorsey, Songhua Xu, Gabe Smedresman, Holly Rushmeier, and Leonard McMillan. The mental canvas: A tool for conceptual architectural design and analysis. In Proc. Pacific Conference on Computer Graphics and Applications, pages 201-210. IEEE Computer Society, October 2007.

8. Tovi Grossman, Ravin Balakrishnan, Gordon Kurtenbach, George Fitzmaurice, Azam Khan, and Bill Buxton. Creating principal 3D curves with digital tape drawing. In CHI '02: Proc. SIGCHI conference on Human factors in computing systems, pages 121-128. ACM, 2002.

9. Martin Hachet and Pascal Guitton. The CAT - when mice are not enough. In Proc. IEEE VR 2004 Workshop: Beyond Glove and Wand Based Interaction, pages 6669, March 2004. Immersion S.A.S. http://www.immersion.fr.

10. T. Igarashi, S. Matsuoka, and H. Tanaka. Teddy: a sketching interface for 3D freeform design. In SIGGRAPH '99: Proc. annual conference on Computer graphics and interactive techniques, pages 409-416. ACM/Addison-Wesley Publishing Co., 1999.

11. O. Karpenko, J. Hughes, and R. Raskar. Free-form Sketching with Variational Implicit Surfaces. Computer Graphics Forum (Proc. Annual Eurographics Conference 2002), 21(3):585-594, 2002.

12. Paul Lapides, Ehud Sharlin, Mario Costa Sousa, and Lisa Streit. The 3D tractus: A three-dimensional drawing board. In IEEE International Workshop on Horizontal Interactive Human-Computer Systems (TableTop '06), January 2006.

13. Florian Levet, Xavier Granier, and Christophe Schlick. Multi-view sketch-based freeform modeling. In International Symposium on Smart Graphics, 2007.

14. Lee Markosian, Jonathan M. Cohen, Thomas Crulli, and John F. Hughes. Skin: A constructive approach to modeling free-form shapes. In SIGGRAPH '99: Proc. annual conference on Computer graphics and interactive techniques, pages 393-400. ACM, August 1999.

15. Andrew Nealen, Takeo Igarashi, Olga Sorkine, and Marc Alexa. Fibermesh: designing freeform surfaces with 3D curves. ACM Trans. Graph., 26(3):41, 2007.

16. S. Owada, F. Nielsen, K. Nakazawa, and T. Igarashi. A Sketching Interface for Modeling the Internal Structures of 3D Shapes. In Proc. Smart Graphics, pages 49-57. Springer-Verlag, July 2003.

17. Emanuel Sachs, Andrew Roberts, and David Stoops. 3-Draw: A tool for designing 3D shapes. IEEE Comput. Graph. Appl., 11(6):18-26, 1991.

18. R. Schmidt, B. Wyvill, M.C. Sousa, and J.A. Jorge. ShapeShop: Sketch-Based Solid Modeling with BlobTrees. In Proc. Eurographics Workshop on Sketch-Based Interfaces and Modeling, pages 53-62, 2005. 
19. Mark Schroering, Cindy Grimm, and Robert Pless. A new input device for 3D sketching. In Vision Interface, pages 311-318, 2003.

20. C.-L. Tai, H. Zhang, and C.-K. Fong. Prototype Modeling from Sketched Silhouettes based on Convolution Surfaces. Computer Graphics Forum, 23(1):71-83, 2004.

21. Steve Tsang, Ravin Balakrishnan, Karan Singh, and Abhishek Ranjan. A suggestive interface for image guided 3D sketching. In CHI '04: Proc. SIGCHI conference on Human factors in computing systems, pages 591-598. ACM, 2004.

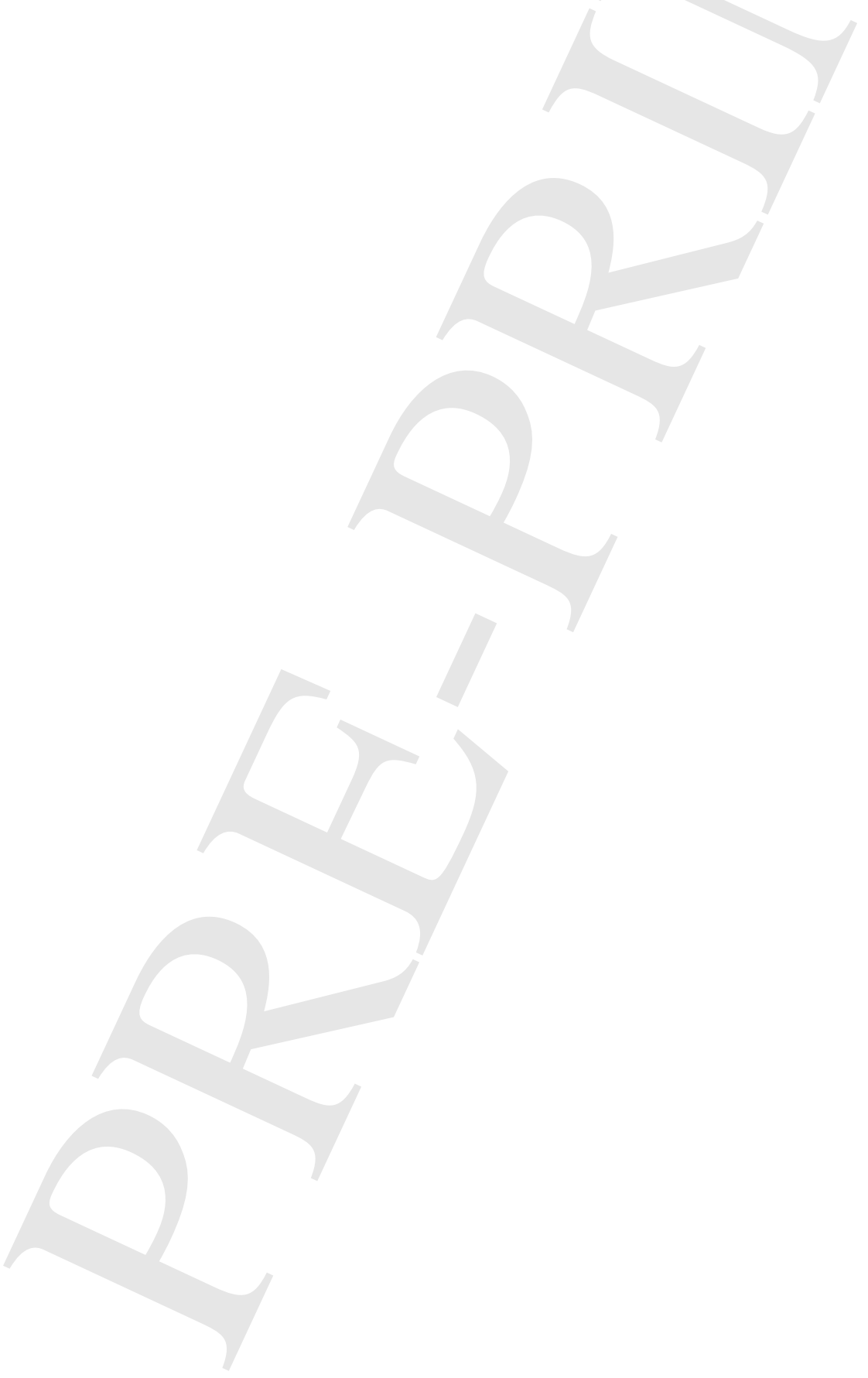

